\title{
Effectiveness and cost-effectiveness of community singing on mental health-related quality of life of older people: randomised controlled trial
}

\author{
Simon Coulton, Stephen Clift, Ann Skingley and John Rodriguez
}

\section{Background}

As the population ages, older people account for a greater proportion of the health and social care budget. Whereas some research has been conducted on the use of music therapy for specific clinical populations, little rigorous research has been conducted looking at the value of community singing on the mental health-related quality of life of older people.

\section{Aims}

To evaluate the effectiveness and cost-effectiveness of community group singing for a population of older people in England.

\section{Method}

A pilot pragmatic individual randomised controlled trial comparing group singing with usual activities in those aged 60 years or more.

\section{Results}

A total of 258 participants were recruited across five centres in East Kent. At 6 months post-randomisation, significant differences were observed in terms of mental health-related quality of life measured using the SF12 (mean difference $=2.35 ; 95 \% \mathrm{Cl}=0.06-4.76$ ) in favour of group singing. In addition, the intervention was found to be marginally more cost-effective than usual activities. At 3 months, significant differences were observed for the mental health components of quality of life (mean difference $=4.77 ; 2.53-7.01$ ), anxiety (mean difference $=-1.78 ;-2.5$ to -1.06 ) and depression (mean difference $=-1.52 ;-2.13$ to -0.92 ).

\section{Conclusions}

Community group singing appears to have a significant effect on mental health-related quality of life, anxiety and depression, and it may be a useful intervention to maintain and enhance the mental health of older people.

\section{Declaration of interest}

S. Clift is a board member of Sing For Your Life Ltd, a notfor-profit third sector organisation which played a role in developing and implementing the intervention reported.

\section{Copyright and usage}

(c) The Royal College of Psychiatrists 2015.
An increasing number of older people in the United Kingdom (UK) account for a significant proportion of health and social care service use, ${ }^{1}$ and this requires novel approaches to maintain and promote mental and physical health of this population. There is evidence that maintaining an active lifestyle mentally, physically and socially is important in contributing to 'successful ageing', well-being and the ability to remain living independently. ${ }^{2}$ Previous evidence-based reviews of interventions aimed at maintaining or enhancing mental health and health-related quality of life in older people found little evidence for a variety of group-based interventions including exercise, t'ai chi and reminiscence groups, ${ }^{3}$ but a systematic review by Bridle et $a l^{4}$ highlighted the benefits of tailored exercise in reducing depression in older people. Recent years have witnessed a growing recognition of the value of participatory arts activities in improving the mental health and overall well-being of older people. ${ }^{5}$ Within the area of music, previous research with older people focused upon the effect of music listening ${ }^{6,7}$ or music therapy, ${ }^{8}$ rather than music as a part of everyday experience. ${ }^{9}$ Clift et $a l^{10}$ conducted a systematic mapping of non-clinical research studies focusing specifically on participatory singing and found only two studies, using standardised measures within controlled trials for older people. ${ }^{5,11}$ Both studies identified improvements in mental health for participants in singing groups, but have serious methodological limitations in terms of lack of justification for sample size and failure to randomise to intervention or control group, issues that limit the value of the evidence. Further reviews have pointed to the value of singing as a therapeutic intervention for older people with long-term physical health conditions, ${ }^{12,13}$ including small pilot randomised controlled trials of singing lessons for people with Chronic Obstructive Pulmonary Disease in clinical settings. ${ }^{14,15}$

The focus of the current study is the evaluation of an innovative community singing initiative, the 'Silver Song Club Project', which provides opportunities for older people to come together to sing with the support of professional musicians. Some 40 such clubs currently exist, mainly in South East England, which are managed by a third sector organisation, Sing for Your Life Ltd. Initially we completed a qualitative, process-orientated evaluation which suggested potential positive benefits across psychological, cognitive, social and physical domains, ${ }^{16}$ and this provided a basis upon which to develop a randomised controlled trial to evaluate the effectiveness and cost-effectiveness of community singing on the mental and physical health-related quality of life of older people.

\section{Aims of the study}

(a) To assess the effectiveness of active engagement in community singing on measures of mental and physical health-related quality of life, depression and anxiety for older people.

(b) To evaluate the cost-effectiveness of active engagement in community singing for older people.

\section{Hypotheses}

Primary hypothesis, stated as null hypothesis: singing groups for older people are no more effective than usual activities in increasing mental health-related quality of life in older people 
measured 6 months after randomisation assessed by the York SF12.

Secondary hypotheses, stated as null hypotheses:

(a) singing groups for older people are no better at reducing anxiety and depression when compared with usual activities at 6 months after randomisation assessed by the Hospital Anxiety and Depression Scale;

(b) singing groups for older people are no more effective than usual activities in increasing physical health-related quality of life in older people assessed 6 months after randomisation by the York SF12; and

(c) singing groups for older people are no more cost-effective than usual activities.

\section{Method}

A prospective, pilot, pragmatic, randomised, controlled trial in which eligible, consenting participants were randomised with equal probability to either singing group participation or usual activities. Randomisation was conducted by a secure remote randomisation service independent of the research team. Randomisation employed random permuted blocks of variable length and was stratified by centre and gender. Participants were followed-up at 3 and 6 months by post. The study was approved by the Surrey NHS ethics committee (ref: 10/H1109/5) and registered (ISRCTN62404401). The study was undertaken in accordance with the Declaration of Helsinki.

\section{Sample size}

The primary outcome measure was the mental health component of quality of life measured by the SF12 at 6 months post-randomisation. A clinically important difference on this dimension is estimated as a difference of five points between intervention and control group, equivalent to a medium effect size difference of $0.5{ }^{17}$ To detect this difference using a two-tailed test, alpha of 0.05 and power at $80 \%$ requires 63 participants in each of the two arms, a total of 126 participants. We anticipated five singing groups and five controls, and had to take account of any clustering effect in calculating sample size. We used a conservative estimate of intra-class correlation coefficient of 0.02 , similar to other community-dwelling older people and a harmonic mean cluster size of 12 . This inflated the required sample size by a factor of 1.2, a total of 154 participants. Previous research suggested that the loss to follow-up at 6 months for this population would be in the order of $20 \%$, and this further inflated the sample size to 184, 92 participants in each of the intervention and control groups.

\section{Participants}

As the intervention focused on maintaining or enhancing the mental health status, experiencing current mental health issues was not a specific inclusion criteria. The study was publicised widely within the local areas. Researchers attended day centres and other venues where older people met for group activities to provide information on the study. In addition, advertisements were placed in the local media, general practices and community venues. Inclusion and exclusion criteria were kept to a minimum to maximise the generalisability of the population. All those expressing an interest and aged 60 years or more were eligible to participate. Only those unable to provide informed consent were excluded.

\section{Procedure}

All individuals indicating an interest in taking part in the study were sent a baseline questionnaire, an information sheet outlining the purpose of the study and a consent form to be returned with the questionnaire. Eligible and consenting participants were randomised to either a singing group or usual activities.

\section{Intervention}

\section{control group}

Individuals in the control group continued with their normal activities. To address any potential resentful demoralisation, members of the control group were informed that they would be welcome to join a singing group at the end of the research study, after completion of the primary outcome assessment at 6 months.

\section{Intervention group}

The Silver Song Club model is an established format for participative singing for older people that was selected for inclusion as one out of three UK examples of good practice for the Health Pro Elderly international project. ${ }^{18}$ Details are available on the Sing for Your Life Ltd website (www.singforyourlife.org.uk/ sites/default/files_new/SSC\%20FormativevEvalulation\%20Summary. pdf). Trained and experienced facilitators under the guidance of Sing for Your Life Ltd met to compile a 14-week 90-minute programme comprising songs from different eras and a variety of genres. This was followed by a series of 'unification' meetings, to ensure that all facilitators were aware of how to access the material and deliver it in the same way (e.g. accompaniment, musical key and acquiring copyright). The programme was developmental, progressing from singing melody lines to harmonising, layering and singing in rounds. Chime bars were also introduced where appropriate, and there was an opportunity for participants to request particular songs. All clubs delivered the same programme concurrently, and at the end of the 14 weeks the clubs disbanded. A programme manager, who made unannounced visits to each group during the intervention period, monitored fidelity. A songbook was produced for the trial and a register of attendees was maintained.

\section{Study measures}

\section{Primary outcome}

The primary outcome measure was mental health-related quality of life assessed by the York SF $12^{19}$ at 6 months postrandomisation. The SF12 contains 12 items addressing both mental and physical health components of quality of life and has established psychometric properties including reliability, validity and sensitivity to change.

\section{Secondary outcome measures}

The SF12 was also used to generate physical health-related components of quality of life. Anxiety and depression were measured using the Hospital Anxiety and Depression Scale. ${ }^{20}$ This scale is validated for community samples and provides both an increasing severity score ranging from 1 to 21 , with higher scores indicating greater severity.

\section{Economic outcome measures}

Health utility was measured by the EQ5D. ${ }^{21}$ This is a short, fivedimensional instrument with three levels which allows the generation of quality-adjusted life years (QALYs). It is routinely 
used in the economic evaluation of healthcare and recommended for cost-effectiveness analyses. Health and social care service utilisation was measured by a specially designed service use postal questionnaire used previously in a number of evaluations including older people $e^{22,23}$ and measures units of health and social care resources including general practice visits, social care involvement, in-patient stays and out-patient attendance.

All outcomes were measured at baseline, before randomisation and then at 3 and 6 months by post. If a participant failed to respond to a follow-up questionnaire, a reminder and an additional questionnaire were sent 4 weeks after the scheduled follow-up date. In addition, we collected process measures consisting of individual attendance at singing groups and fidelity information on the delivery of singing groups.

\section{Analysis}

As a study of effectiveness, the primary analysis was by intentionto-treat where participants were analysed as part of their allocated group irrespective of the actual treatment received. The primary outcome measure, SF12 mental components at 6 months, was analysed by an analysis of covariance adjusting for baseline age and gender which are known covariates. As the intervention involved groups, we adjusted the analysis using the Huber-White sandwich estimation technique to generate robust standard errors. Secondary outcomes were analysed in a similar manner.

The incremental cost-effectiveness of singing groups compared with usual activities was assessed from a health and social care perspective in accordance with the National Institute of Clinical Excellence guidelines. ${ }^{24}$ The costs associated with setting up and running singing groups were assessed from the actual local costs including the cost associated with premises and managerial overheads. Units of service utilisation in the 6 months before and 6 months after randomisation were assessed from the service use questionnaire, and the net costs for each arm of the study were derived by multiplying these by national sources of unit costs, ${ }^{25}$ as all costs were collected within a 12-month period and no discounting was applied. The EQ5D was used with population values to calculate the QALY change using the area under the curve method. ${ }^{26}$ As economic data tend to be skewed, we used an established bootstrapping technique, resampling with replacement, to derive more robust confidence interval estimates. ${ }^{27}$ We divided the differences in the net costs for each arm by the difference in QALY gains to yield an incremental cost-effectiveness ratio. We estimated the sampling distribution from 1000 bootstrapped samples and derived the cost-effectiveness acceptability curves. ${ }^{28}$ These curves plot the resulting probability that one arm is better than the other, against the maximum policy-makers may be willing to pay for an additional QALY.

\section{Results}

\section{Sample characteristics}

Recruitment to the study took place across five localities in East Kent. A total of 393 potential participants expressed an interest to participate and were sent information on the study and a baseline questionnaire. Of these only 258 (66\%) were eligible and consented to participate in the study, 127 (49\%) were allocated to the control and $131(51 \%)$ were allocated to the intervention. Follow-up rates at 3 and 6 months were $222(86 \%)$ and 204 (79\%), respectively, and no differential follow-up rate between the groups was observed. Of those allocated to the singing groups, $106(81 \%)$ attended at least $50 \%$ of the sessions and attendance was similar across all centres. A full CONSORT diagram is provided in Fig. 1.

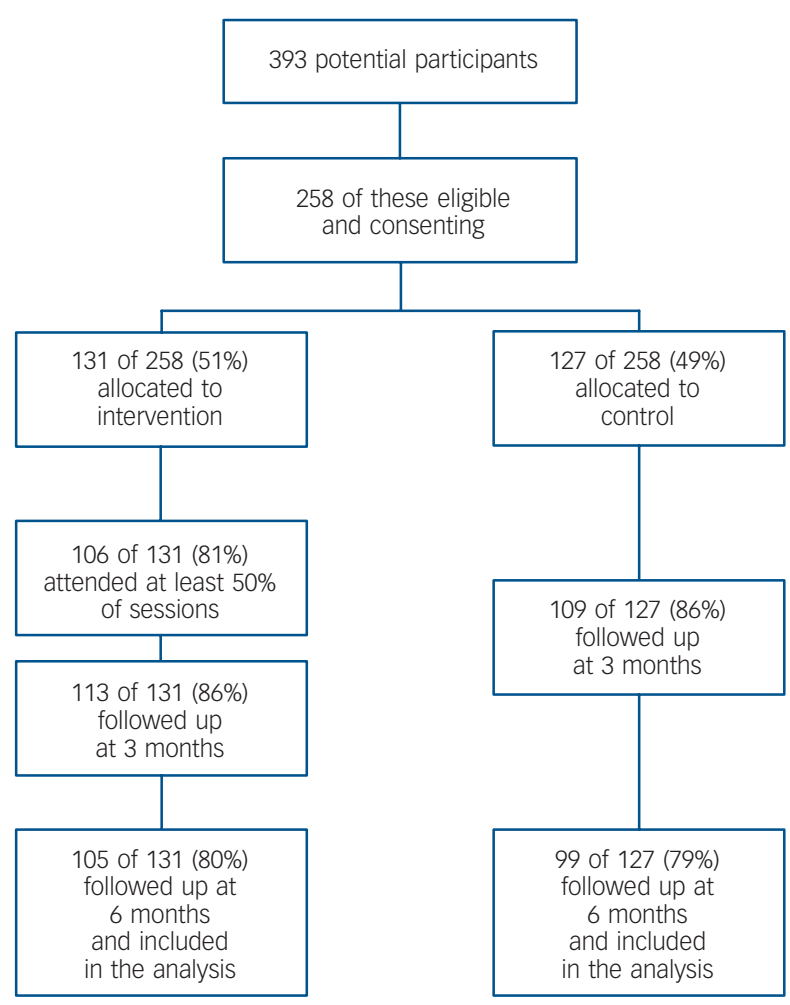

Fig. 1 Trial CONSORT statement

Baseline demographics and outcome measures are provided in Table 1. The mean age was 69 years (s.d. 7.14); the majority were female (84\%) and White (98\%). No statistical differences in baseline demographics or baseline outcome measures were observed between the groups.

\section{Primary outcome}

At baseline, SF12 mental health-related quality of life was similar across the groups, with 50.0 (95\% CI 47.9-52.2) and 48.8 (46.850.8 ) for the control and intervention groups, respectively. Although these were similar in the control group at 6 months, 49.9 (48.2-51.7), they had improved in the intervention group,

\begin{tabular}{|lccc|}
\hline Table 1 Baseline description of the sample & \\
& $\begin{array}{c}\text { Overall } \\
(n=258)\end{array}$ & $\begin{array}{c}\text { Control } \\
(n=127)\end{array}$ & $\begin{array}{c}\text { Intervention } \\
(n=131)\end{array}$ \\
\hline Demographics & & & \\
Mean age (s.d.) & $69.2(7.14)$ & $69.5(7.13)$ & $69.2(7.18)$ \\
Female $n$ (\%) & $214(83.9)$ & $108(87.1)$ & $106(80.9)$ \\
Smoking $n$ (\%) & $11(4.3)$ & $3(2.4)$ & $8(6.2)$ \\
White $n$ (\%) & $250(98.0)$ & $120(96.8)$ & $130(99.2)$ \\
Employed $n$ (\%) & $25(11.0)$ & $9(8.1)$ & $16(13.8)$ \\
Education after 16 $n$ (\%) & $162(62.8)$ & $79(64.8)$ & $83(63.8)$ \\
\hline Outcome measures & & & \\
Mean SF12 & & & $39.1(6.58)$ \\
- physical score (s.d.) & $39.4(6.63)$ & $39.8(6.69)$ & \\
Mean SF12 & & & \\
- mental Score (s.d.) & $49.4(11.7)$ & $50.0(11.9)$ & $48.8(11.5)$ \\
Mean EQ5D score (s.d.) & $0.74(0.22)$ & $0.74(0.22)$ & $0.74(0.22)$ \\
Mean HADS - anxiety (s.d.) & $6.40(4.46)$ & $6.41(4.57)$ & $6.40(4.46)$ \\
Anxiety case $n$ (\%) & $49(19.1)$ & $24(19.0)$ & $25(19.1)$ \\
Mean HADS & & & \\
- depression (s.d.) & $4.62(3.52)$ & $4.28(3.52)$ & $4.95(3.52)$ \\
Depression case $n$ (\%) & $20(7.8)$ & $8(6.3)$ & $12(9.2)$ \\
\hline
\end{tabular}




\begin{tabular}{|c|c|c|c|c|c|c|c|}
\hline & \multicolumn{2}{|l|}{ Baseline } & \multicolumn{3}{|l|}{ Month 3} & \multicolumn{2}{|l|}{ Month 6} \\
\hline & $\begin{array}{c}\text { Mean } \\
(95 \% \mathrm{Cl})\end{array}$ & $\begin{array}{l}\text { Mean } \\
(95 \% \mathrm{Cl})\end{array}$ & $\begin{array}{l}\text { Mean difference } \\
\qquad(95 \% \mathrm{Cl})\end{array}$ & $P$ & $\begin{array}{c}\text { Mean } \\
(95 \% \mathrm{Cl})\end{array}$ & $\begin{array}{l}\text { Mean difference } \\
\qquad(95 \% \mathrm{Cl})\end{array}$ & $P$ \\
\hline \multicolumn{8}{|l|}{ SF12 - physical } \\
\hline Control & $39.8(38.6-40.9)$ & $39.2(38.3-40.0)$ & & & $39.6(38.6-40.7)$ & & \\
\hline Intervention & $39.1(37.9-40.3)$ & $40.0(39.1-40.8)$ & $0.83(-0.39-2.05)$ & 0.18 & $39.9(38.7-40.9)$ & $0.26(-1.75-1.23)$ & 0.73 \\
\hline \multicolumn{8}{|l|}{ SF12 - mental } \\
\hline Control & $50.0(47.9-52.2)$ & $50.7(49.1-52.3)$ & & & $49.9(48.2-51.7)$ & & \\
\hline Intervention & $48.8(46.8-50.8)$ & $55.5(53.9-57.1)$ & $4.77(2.53-7.01)$ & $<0.01$ & $52.3(50.7-54.0)$ & $2.35(0.06-4.76)$ & 0.05 \\
\hline \multicolumn{8}{|l|}{ HADS - anxiety } \\
\hline Control & $6.41(5.62-7.20)$ & $6.01(5.41-6.42)$ & & & $5.83(5.30-6.36)$ & & \\
\hline Intervention & $6.40(5.62-7.18)$ & $4.14(3.64-4.64)$ & $-1.78(-2.50-1.06)$ & $<0.01$ & $5.26(4.75-5.76)$ & $-0.57(-1.31-0.16)$ & 0.13 \\
\hline \multicolumn{8}{|c|}{ HADS - depression } \\
\hline Control & $4.28(3.67-4.89)$ & $4.15(3.72-4.56)$ & & & $4.22(3.71-4.73)$ & & \\
\hline Intervention & $4.95(4.53-5.57)$ & $2.63(2.21-3.05)$ & $-1.52(-2.13-0.92)$ & $<0.01$ & $3.69(3.20-4.18)$ & $-0.53(-1.24-0.18)$ & 0.14 \\
\hline \multicolumn{8}{|l|}{ EQ5D - QALY } \\
\hline Control & $0.76(0.72-0.81)$ & $0.78(0.74-0.82)$ & & & $0.77(0.72-0.82)$ & & \\
\hline Intervention & $0.76(0.71-0.80)$ & $0.80(0.76-0.85)$ & $0.02(0.01-0.03)$ & 0.05 & $0.78(0.73-0.83)$ & $0.01(0.01-0.02)$ & 0.01 \\
\hline
\end{tabular}

52.3 (50.7-54.0). The mean difference between intervention and control at 6 months was $2.35(0.06-4.76)$, and this was significant $(P=0.05$; Table 2).

\section{Secondary outcomes}

At 6 months, no significant differences were observed between the groups in terms of SF12 physical components of health-related quality of life, anxiety or depression (Table 2). At 3 months, significant differences between the groups were observed in terms of mental components of SF12 health-related quality of life and mean difference intervention compared with control 4.77 (2.53$7.01)$, anxiety $-1.78(-2.50$ to -1.06$)$ and depression -1.52 $(-2.13$ to -0.92$)$. No other significant differences were observed between the groups at 3 months (Table 2).

\section{Economic outcomes}

The costs of implementing and training staff to conduct the singing groups are shown in Table 3 . Training costs were estimated over a 12-month period where the average facilitator would deliver 80 sessions, two per week, to avoid an overestimation of training costs. The total cost per session was estimated at $£ 176.84$ and the cost per participant over 14 sessions was estimated at $\mathfrak{k} 18.88$.

Service use was measured at baseline and 6 months, and costs of units consumed were derived from national sources. Service use costs were estimated only for those who were followed-up and are presented in Table 4. Service use costs increased in both groups between baseline and 6 months, but although the increase was

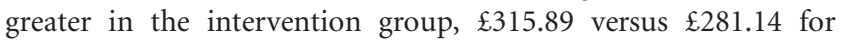
the control group, this difference was not significant. Participants in the control group gained 0.008 QALYs between baseline and 6 months compared with a gain of 0.023 QALYs in the intervention group, the difference between the groups of 0.015 (95\% CI 0.014-0.016) was significant.

A cost-effectiveness acceptability curve was constructed (Fig. 2). This indicated that at a willingness to pay threshold of zero, the control group would be the preferred economic option. At a willingness to pay threshold of $£ 20000$, the intervention has a $60 \%$ probability of being the more cost-effective option, and at recommended willingness to pay thresholds of $£ 30000$ (24), this probability increases to $64 \%$.
Table 3 Implementation and training costs associated with singing groups

\begin{tabular}{|c|c|c|}
\hline Resource & $\begin{array}{l}\text { Unit cost } \\
\text { per session } \\
\text { (f) }\end{array}$ & $\begin{array}{c}\text { Cost per } \\
\text { participant } \\
(n=131)\end{array}$ \\
\hline $\begin{array}{l}\text { Training costs } \\
\text { Facilitators ( } 5 \text { facilitators, } \\
3 \text { days at } £ 75 / \text { day) } \\
\text { Facilitator expenses ( } 5 \text { facilitators, } \\
3 \text { journeys at } £ 35 / j o u r n e y) \\
\text { Trainer ( } 3 \text { days at } £ 100 / \text { day) } \\
\text { Trainer expenses } \\
\text { ( } 3 \text { journeys at } £ 35 / \text { journey) } \\
\text { Venue hire ( } 3 \text { days @ £100/day) }\end{array}$ & $\begin{array}{l}0.26^{\mathrm{d}} \\
0.75^{\mathrm{e}}\end{array}$ & $\begin{array}{l}0.14 \\
0.08\end{array}$ \\
\hline $\begin{array}{l}\text { Capital expenditure } \\
\text { Hand chimes } \\
\text { Keyboard } \\
\text { Song sheets }\end{array}$ & $\begin{array}{l}1.25^{f} \\
1.75^{g} \\
0.22^{h}\end{array}$ & $\begin{array}{l}0.13 \\
0.19 \\
0.02\end{array}$ \\
\hline $\begin{array}{l}\text { Indirect cost } \\
\text { Advertising } \\
\text { Management } \\
\text { Administration }\end{array}$ & $\begin{array}{r}2.14^{i} \\
9.64^{j} \\
12.86^{k}\end{array}$ & $\begin{array}{l}0.23 \\
1.03 \\
1.37 \\
\end{array}$ \\
\hline $\begin{array}{l}\text { Session costs } \\
\text { Facilitator } \\
\text { Facilitator expenses } \\
\text { Venue hire } \\
\text { Refreshments }\end{array}$ & $\begin{array}{r}75.00^{\prime} \\
35.00^{\prime} \\
30.00^{\prime} \\
3.00^{\prime}\end{array}$ & $\begin{array}{l}8.01 \\
3.74 \\
3.21 \\
0.32\end{array}$ \\
\hline Total & 176.84 & 18.88 \\
\hline \multicolumn{3}{|c|}{ 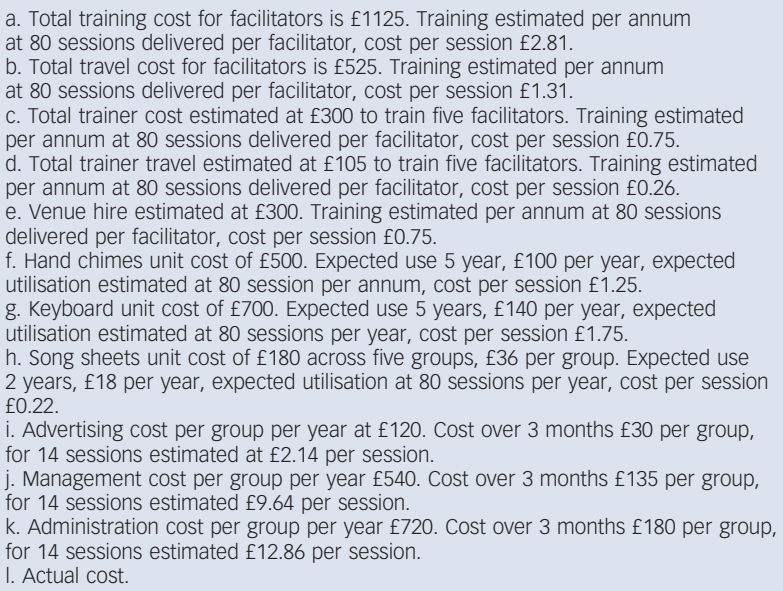 } \\
\hline
\end{tabular}




\begin{tabular}{|c|c|c|c|c|}
\hline & Social care $(f)$ & Primary care $(\mathrm{f})$ & Secondary care $(£)$ & Total (f) \\
\hline \multicolumn{5}{|l|}{ Baseline } \\
\hline Control & $2.58(1.57)$ & $66.38(7.83)$ & 273.62 (64.70) & 342.59 (67.39) \\
\hline Intervention & $4.06(2.23)$ & $60.45(5.51)$ & 229.58 (50.39) & 294.09 (52.87) \\
\hline \multicolumn{5}{|l|}{ Month 6} \\
\hline Control & $5.04(3.05)$ & $85.21(8.66)$ & 533.48 (126.91) & $623.73(131.16)$ \\
\hline Intervention & $3.24(1.82)$ & $78.16(8.25)$ & $528.58(208.70)$ & $609.98(210.15)$ \\
\hline
\end{tabular}

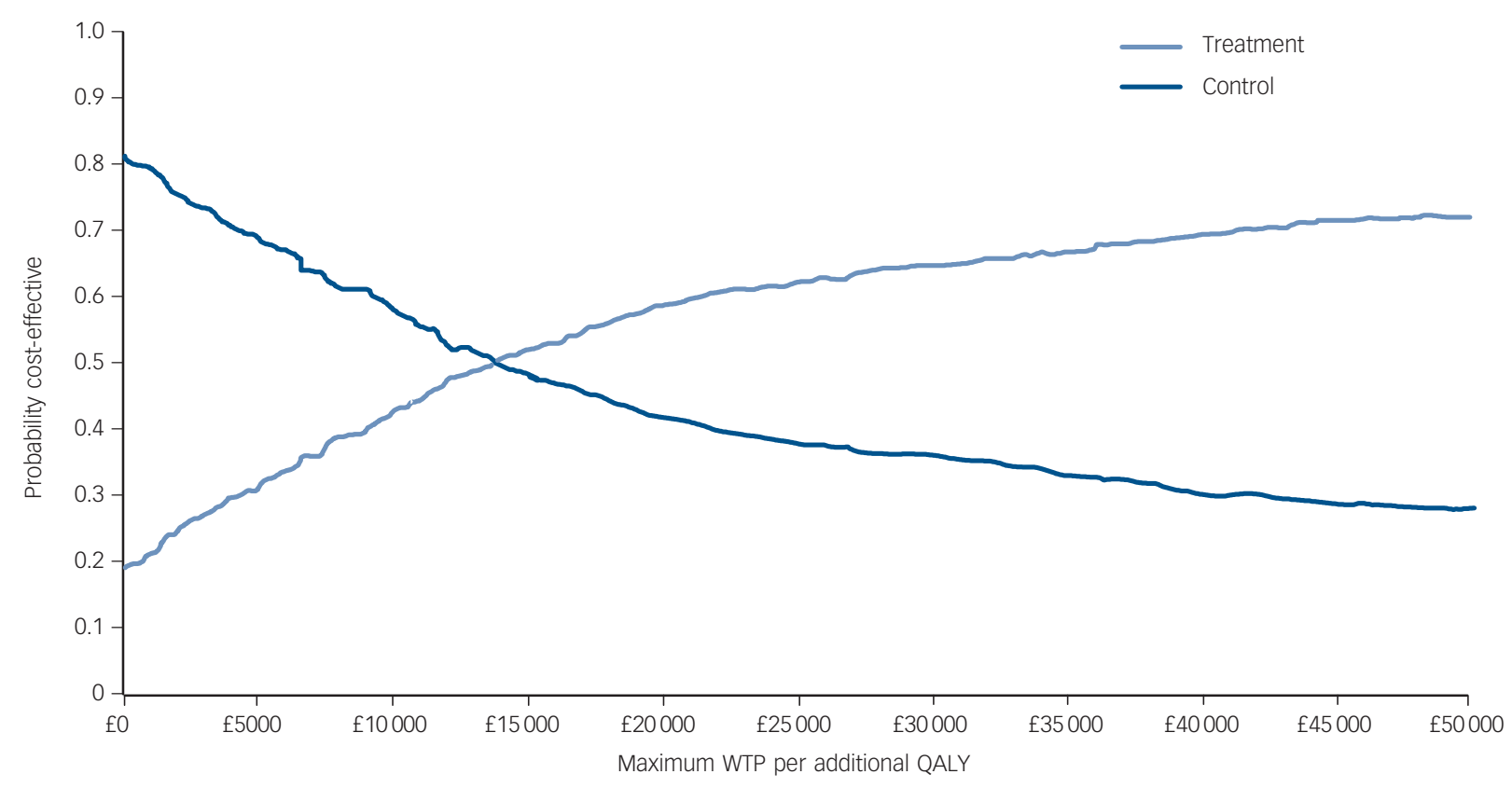

Fig. 2 Cost-effectiveness acceptability curve comparing the probability of cost-effectiveness for intervention and control at different QALY valuations.

\section{Discussion}

The reported study is the first pragmatic randomised controlled trial of community singing groups for older people, focusing on their mental health and quality of life. The interest shown in the groups and the willingness of participants to engage in singing groups is a clear indication of both feasibility and acceptability of community singing for the older people. Our primary hypothesis explored the potential benefits of singing groups on maintaining the mental health-related quality of life of older people. The results suggest that participation in singing groups confers significant benefits in terms of mental aspects of quality of life derived using SF12 and appears cost-effective when compared to usual activities 6 months after randomisation and 3 months after the groups had ceased to meet. No differences were observed at 6 months in terms of physical aspects of quality of life, anxiety or depression. At 3 months, at the end of the intervention, levels of anxiety and depression were significantly lower in the singing group. This suggests that the greatest benefit occurs when participants are engaged in singing groups and continued access to singing groups may confer important benefits on the mental health of the older population.

Qualitative feedback from participants through written comments and interviews was highly positive. People indicated their enjoyment of the experience and highlighted the benefits on mental health, well-being and social relationships.
A clear marker of the value participants placed on the singing groups is the fact that four of the five groups established for research purposes were reinstated at the end of the 6 month follow-up and continue to meet and have grown in membership with support from a new charity Living Lively (http://www. livinglively.org.uk/). The study adds weight to the notion that meaningful, social and pleasurable activities can confer mental health benefits to participants identified in other studies of music therapy. ${ }^{8}$

In conclusion, the provision of opportunities to meet and sing together provide an opportunity to maintain and enhance the mental health of older people that is cost-effective and acceptable to the population, and should be considered as an important element in any public mental health strategy for this population.

Limitations of our study include the fact that it was conducted in one geographical area where the population is predominantly White British. We do not know, therefore, whether our findings could be generalised to other areas with different demographic characteristics. The groups also ran for a relatively short period of time and it is possible that longer involvement in singing could lead to more substantial and sustained benefit. As the study reported was pragmatic, we did not explore in detail the processes of change that may underpin any observed changes and understanding these processes may be important in understanding the relationship between group musical activity and improvements in well-being.

It may be the case that any group activity confers similar benefits and that singing groups are just one form of group 
activity and further research is needed to address the relative effects of group singing versus other group activities. Yet it is important to note the ease with which the sample was recruited and the high levels of engagement would suggest that group singing is both feasible and acceptable to older people. In addition, the design of the study involved a waiting list control group, where participants allocated to the control group had the intervention made available at the end of the study. The reasons for this involved addressing an issue of resentful demoralisation. It may have been the case that the control group was perceived as a delayed intervention for some participants and this may have impacted on their reporting of the outcomes. If this was the case, then the reported effects at 6 months may have been underestimates of the true effect. In addition, it is important to be clear that the sample was a self-selecting population, people who wanted to engage in singing groups, but the ease of recruitment and the numbers of expressions of interest suggest there are large numbers of individuals who would like to engage in singing groups if they were more widely available.

Further, as our focus was on mental health-related quality of life, the study population was not specifically experiencing severe mental health issues. We have undertaken an observational study of group singing for people with enduring and severe mental health issues over a year, and this demonstrated clinically important improvement in mental health outcomes. The next step in building on the pilot trial reported here should be a larger scale multi-centre trial running over a longer period of time.

\section{Funding}

This is a summary of independent research funded by a National Institute for Health Research (NIHR) Research for Patient Benefit grant. The views expressed are those of the authors and not necessarily those of the NHS, the NIHR or the Department of Health.

\section{Acknowledgements}

We thank our collaborators and all the participants who contributed to the conduct of this study.

Simon Coulton, MSc, Centre for Health Service Studies, University of Kent, Canterbury; Stephen Clift, PhD, FRSPH, Sidney De Haan Research Centre for Arts and Health, Canterbury Christ Church University, Canterbury; Ann Skingley, PhD, RN, Sidney De Haan Research Centre for Arts and Health, Canterbury Christ Church RN, Sidney De Haan Research Centre for Arts and Health, Canterbury Christ Church
University, Canterbury; John Rodriguez, MBMA, MRCP, MFPM, FFPH, NHS Kent and Medway, Ashford, UK

Correspondence Simon Coulton, Centre for Health Service Studies, University of Kent, Canterbury CT2 7NZ, UK. Email: s.coulton@kent.ac.uk

First received 29 Mar 2013, final revision 5 Nov 2014, accepted 6 Nov 2014

\section{References}

1 Imison C. Future Trends. Kings Fund, 2012.

2 Health Development Agency. Taking Local Action: Improving the Health and Wellbeing of People in Mid-life and Beyond. NICE, 2004

3 Van Malderen L, Mets T, Gorus E. Interventions to enhance the Quality of Life of older people in residential long-term care: a systematic review. Ageing Res Rev 2013; 12: 141-50.
4 Bridle C, Spanjers K, Patel S, Atherton NM, Lamb SE. Effect of exercise on depression severity in older people: systematic review and meta-analysis of randomised controlled trials. Br J Psychiatry 2012; 201: 180-5.

5 Cohen GD, Perlstein S, Chapline J, Kelly J, Firth KM, Simmens S. The impact of professionally conducted cultural programs on the physical health, mental health, and social functioning of older adults. Gerontologist 2006; 46: $726-34$.

6 Coffman D. Music and quality of life in older people. Psychomusicology 2006; 46: $76-88$.

7 Stacey R, Brittain K, Kerr S. Singing for health: an exploration of the issues. Health Educ 2002; 199: 132-29.

8 Erkkila J, Punkanen M, Fachner J, Ala-Ruona E, Pontio I, Tervaniemi M, et al. Individual music therapy for depression: randomised controlled trial. Br J Psychiatry 2011; 199: 132-9.

9 Sixsmith A, Gibson G. Music and the wellbeing of people with dementia Ageing Soc 2006; 27: 127-45.

10 Clift S, Nicols J, Raisbeck M, Whitmore C, Morrison I. Group singing, wellbeing and health: a systematic mapping of the research evidence. UNESCO J 2010; 2: 1-25

11 Houston D, McKee J, Carroll L, Marsh $\mathrm{H}$. Using humour to promote psychological wellbeing in residential homes for older people. Ageing Mental Health 1998; 2: 328-32.

12 Clark I, Harding K. Psychosocial outcomes of active singing as a therapeutic intervention: a review of the literature. Nordic J Mus Ther 2012; 21: 80-98.

13 Gick M. Singing, health and wellbeing: a health psychologist's review. Psychomusicology 2011; 21: 176-207.

14 Lord VM, Cave P, Hume VJ, Flude EJ, Evans A, Kelly JL, et al. Singing teaching as a therapy for chronic respiratory disease - a randomised controlled trial and qualitative evaluation. BMC Pulm Med 2010; 10: 41.

15 Lord VM, Hume VJ, Kelly JL, Cave P, Silver J, Waldman M, et al. Singing classes for chronic obstructive pulmonary disease: a randomized controlled trial. BMC Pulm Med 2012; 12: 69.

16 Skingley $A$, Bungay $H$. The Silver Song Club Project: singing to promote the health of older people. Br J Community Nurs 2010; 15: 135-40.

17 Qualymetric. SF12 v2 Health Survey Users Manual. Qalymetric, 2010.

18 Billings J, Brown P. HealthProElderly - Evidence-based Guidelines for Older People: United Kingdom National Evaluation Report. University of Kent, 2008.

19 Iglesias CP, Birks YF, Torgerson DJ. Improving the measurement of quality of life in older people: the York SF-12. QJM 2001; 94: 695-8.

20 Snaith R, Sigmond A. The Hospital Anxiety and Depression Scale. NFER, 1994.

21 EuroQoL Group. EuroQoL - a new facility for the measurement of healthrelated quality of life. Health Pol 1990; 16: 199-208.

22 Richardson G, Bloor K, Williams J, Russell I, Durai D, Cheung WY, et al. Cost effectiveness of nurse delivered endoscopy: findings from randomised multi-institution nurse endoscopy trial (MINUET). BMJ 2009; 338: b270.

23 Watson JM, Crosby H, Dale VM, Tober G, Wu Q, Lang J, et al. AESOPS: a randomised controlled trial of the clinical effectiveness and costeffectiveness of opportunistic screening and stepped care interventions for older hazardous alcohol users in primary care. Health Technol Assessment 2013; 17(25): 1-158.

24 NIHSCE. Guide to the Methods of Technology Appraisal. NIHSCE, 2013.

25 Curtis L. The Unit Costs of Health and Social Care. (ed PSSRU). University of Kent, 2007.

26 Brooks R, Rabin R, de Charro F. The Measurement and Valuation of Health Status using EQ-5D: A European Perspective. Kluwer Academic, 2003.

27 Efron B, Tibshirami R. An Introduction to the Bootstrap. Chapman \& Hall, 1993.

28 Fenwick E, Claxton K, Sculpher M. Representing uncertainty: the role of the cost-effectiveness acceptability curves. Health Econ 2001; 10: 779-87. 